J. Saastamoinen

National Research Council of Canada

ABSTRACT

Approximations have been removed from the derivation of the coefficients in the binomial expansion for astronomical refraction, $\Delta z_{1}=$ $\tan z_{1}\left(Y_{0}-\frac{1}{2} Y_{1} \sec ^{2} z_{1}+\frac{3}{8} Y_{2} \sec ^{4} z_{1}-\ldots\right)$, allowing the calculation of any number of terms to any precision desired. The range of the refraction formula has been extended to greater zenith distances $\left(<90^{\circ}\right)$ by inserting a damping factor into the binomial formula, truncating the expansion at a proper point, and rearranging the terms. Another, computer-manipulated series has been developed for zenith distances at or near the horizon. Further applications include the calculation of photogrametric and parallactic refractions, as well as range corrections in satellite geodesy.

\title{
NOTATION
}

The notation has been taken from an earlier work (Sadstamoinen, 1972-73) on the same subject matter, although important changes and additions have been made to facilitate the derivation of general formulas not presented before. The following symbols appear in the text without explanation:

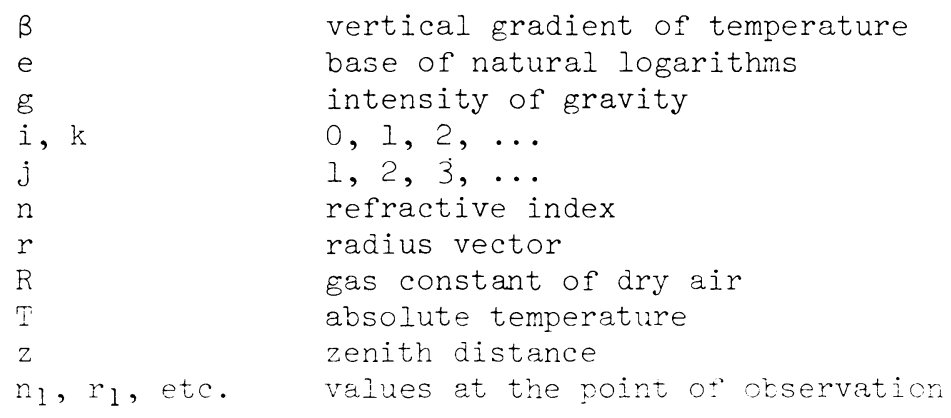




$$
\begin{aligned}
& r_{a}, r_{b} \text {, etc. values at the lower and upper limits, respec- } \\
& \text { tively, of an atmospheric layer with } \beta \text { const. }
\end{aligned}
$$

The same symbol may occasionally be used in different meanings (e also for partial pressure of water vapor) if confusion is unlikely and the notation is familiar from geodetic and meteorological literature.

\section{ASTRONOMICAL REFRACTION}

\subsection{Introduction.}

For the evaluation of astronomical refraction

$$
\Delta z_{1}=\int_{0}^{\log _{e} n_{1}} \tan z \log _{e} n
$$

in a spherically symmetric model atmosphere, the law of refraction

$$
y \sin z=\sin z_{1}
$$

where

$$
\mathrm{y}=\mathrm{nr} /\left(\mathrm{n}_{1} \mathrm{r}_{1}\right)
$$

gives

$$
\begin{aligned}
& \tan z=\tan z_{1}\left[1+\left(y^{2}-1\right) \sec ^{2} z_{1}\right]^{-\frac{1}{2}} \\
& =\tan z_{1}\left[1+(-)^{k} \sum_{k=1}^{\infty} \frac{1 \cdot 3 \cdot 5 \ldots(2 k-1)}{2 \cdot 4 \cdot 6 \ldots(2 k)}\left(y^{2}-1\right)^{k} \sec ^{2 k} z_{1}\right] .
\end{aligned}
$$

Binomial series (3) is convergent if $\left(y^{2}-1\right) \sec ^{2} z_{1}<1$.

Providing that the condition for convergence is satisfied, the contribution to the astronomical refraction of a layer of air between radii vectors $r_{a}$ and $r_{b}$ is consequently

$$
\Delta z_{1}(a ; b)=\tan z_{1}\left(Y_{0}-\frac{1}{2} Y_{1} \sec ^{2} z_{1}+\frac{3}{8} Y_{2} \sec ^{4} z_{1}-\frac{5}{16} Y_{3} \sec ^{6} z_{1}+\ldots\right)
$$

where the coefficients

$$
Y_{k}=\int_{\log _{e} n_{b}}^{\log _{e} n}\left(y^{2}-1\right)^{k} d \log _{e} n
$$

are functions of the model atmosphere employed. Total astronomical 
refraction ( 1 ) is the sum of the contributions from all the layers between the point of observation and the top of the atmosphere.

Power series (4), or the variant

$$
\Delta z_{1}(a ; b)=\tan z_{1}\left(Y_{0}^{\prime}-\frac{1}{2} Y_{1}^{\prime} \tan ^{2} z_{1}+\frac{3}{8} Y_{2}^{\prime} \tan ^{4} z_{1}-\frac{5}{16} Y_{3}^{\prime} \tan ^{6} z_{1}+\ldots\right)
$$

for which binomial series

$$
\begin{aligned}
& \tan z=\frac{1}{y} \tan z_{1}\left[1+\frac{1}{y^{2}}\left(y^{2}-1\right) \tan ^{2} z_{l}\right]^{-\frac{1}{2}} \\
& =\tan z_{1}\left[\frac{1}{y}+(-)^{k} \sum_{k=1}^{\infty} \frac{1 \cdot 3 \cdot 5 \ldots(2 k-1)}{2 \cdot 4 \cdot 6 \ldots(2 k)} \frac{1}{y^{2 k+1}}\left(y^{2}-1\right)^{k} \tan ^{2 k} z_{1}\right]
\end{aligned}
$$

gives the coefficients

$$
Y_{k}^{\prime}=\int_{\log _{e} n_{b}}^{\log _{e} n_{a}}(1 / y)^{2 k+1}\left(y^{2}-1\right)^{k} \log _{e} n,
$$

is the common foundation of most formulas proposed for the calculation of astronomical refraction (Teleki, 1974). Essential differences are found, however, in the way of calculation of the coefficients.

1.2 Calculation of coefficients $\mathrm{Y}_{\mathrm{k}}$ in terms of atmospheric integrals. Multiplication of the identity,

$$
\begin{aligned}
& \mathrm{y}^{2}-1=\frac{2}{\mathrm{n}_{1}{ }^{2} r_{1}}\left(r-r_{1}\right)-\frac{2}{\mathrm{n}_{1}}\left(\mathrm{n}_{1}-\mathrm{n}\right)+\frac{1}{\mathrm{n}_{1}{ }^{2} r_{1}{ }^{2}}\left(r-r_{1}\right)^{2} \\
& +\frac{4}{\mathrm{n}_{1}{ }^{2} r_{1}}(\mathrm{n}-1)\left(r-r_{1}\right)+\frac{1}{\mathrm{n}_{1}{ }^{2}}\left(n_{1}-n\right)^{2}+\frac{2}{\mathrm{n}_{1}{ }^{2} r_{1}{ }^{2}}(n-1)\left(r-r_{1}\right)^{2} \\
& +\frac{2}{\mathrm{n}_{1}{ }^{2} r_{1}}(n-1)^{2}\left(r-r_{1}\right)+\frac{1}{n_{1}{ }^{2} r_{1}{ }^{2}}(n-1)^{2}\left(r-r_{1}\right)^{2}
\end{aligned}
$$

by

$$
\log _{e} n=d n / n=d n-(n-1) d n+(n-1)^{2} d n-(n-1)^{3} d n+\ldots
$$

gives

$$
\begin{aligned}
& \left(y^{2}-1\right) d \log _{e} n=\frac{2}{n_{1}^{2} r_{1}}\left(r-r_{1}\right) d n-\frac{2}{n_{1}}\left(n_{1}-n\right) d \log _{e} n \\
& +\frac{1}{n_{1}{ }^{2} r_{1}{ }^{2}}\left(r-r_{1}\right)^{2} d n+\frac{2}{n_{1}{ }^{2} r_{1}}(n-1)\left(r-r_{1}\right) d n+\frac{1}{n_{1}{ }^{2}}\left(n_{1}-n\right)^{2} d \log _{e} n
\end{aligned}
$$


$+\frac{1}{n_{1}^{2} r_{1}^{2}}(n-1)\left(r-r_{1}\right)^{2} d n$

It is easy to verify that equation (6) is also a mathematical identity.

We can now write, in terms of atmospheric integrals given by the definitions

$$
\begin{aligned}
& P(i, j)=\frac{1}{r_{1}^{j}} \int_{n_{b}}^{n}(n-1)^{i}\left(r-r_{1}\right)^{j} d n \\
& P^{\prime}(i, j)=P(i, j)+\frac{j}{i+1} P(j-l, i+1)
\end{aligned}
$$

and

$$
Q(i)=\int_{\log _{e} n_{b}}^{\log _{e} n_{a}}\left(n_{1}-n\right)^{i} \operatorname{llog}_{e} n,
$$

the formulas

$$
\begin{aligned}
& Y_{0}=Q(0) \\
& n_{1}{ }^{2} Y_{1}=2 P(0,1)-2 n_{1} Q(I)+P^{\prime}(0,2)+Q(2)+P(1,2)
\end{aligned}
$$

for the calculation of the first two coefficients in series expansion (4).

Similarly, by actual multiplication, is obtained the identity

$$
\begin{aligned}
& \left(y^{2}-1\right)^{2} \operatorname{dlog}_{e} n=\frac{4}{n_{1}^{4} r_{1}}\left(1-n_{1}^{2}\right)\left(r-r_{1}\right) d n+\frac{2}{n_{1}^{4} r_{1}^{2}}\left(3-n_{1}^{2}\right)\left(r-r_{1}\right)^{2} d n \\
& +\frac{4}{n_{1}{ }^{4} r_{1}}\left(3-n_{1}^{2}\right)(n-1)\left(r-r_{1}\right) d n+\frac{4}{n_{1}^{2}}\left(n_{1}-n\right)^{2} d \log _{e} n+\frac{4}{n_{1}^{4} r_{1}^{3}}\left(r-r_{1}\right)^{3} d n \\
& +\frac{2}{n_{1}^{4} r_{1}^{2}}\left(9-n_{1}^{2}\right)(n-1)\left(r-r_{1}\right)^{2} d n+\frac{12}{n_{1}^{4} r_{1}}(n-1)^{2}\left(r-r_{1}\right) d n \\
& -\frac{4}{n_{1}{ }^{3}}\left(n_{1}-n\right)^{3} d \log _{e} n+\frac{1}{n_{1}{ }^{4} r_{1}{ }^{4}}\left(r-r_{1}\right)^{4} d n+\frac{12}{n_{1}^{4} r_{1}{ }^{3}}(n-1)\left(r-r_{1}\right)^{3} d n \\
& +\frac{18}{n_{1}^{4} r_{1}^{2}}(n-1)^{2}\left(r-r_{1}\right)^{2} d n+\frac{4}{n_{1}^{4} r_{1}}(n-1)^{3}\left(r-r_{1}\right) d n+\frac{1}{n_{1}^{4}}\left(n_{1}-n\right)^{4} d \log _{e} n \\
& +\frac{3}{n_{1}{ }^{4} r_{1}{ }^{4}}(n-1)\left(r-r_{1}\right)^{4} d n+\frac{12}{n_{1}{ }^{4} r_{1}{ }^{3}}(n-1)^{2}\left(r-r_{1}\right)^{3} d n \\
& +\frac{6}{n_{1}^{4} r_{1}^{2}}(n-1)^{3}\left(r-r_{1}\right)^{2} d n+\frac{3}{n_{1}^{4} r_{1}^{4}}(n-1)^{2}\left(r-r_{1}\right)^{4} d n \\
& +\frac{4}{n_{1}^{4} r_{1}{ }^{3}}(n-1)^{3}\left(r-r_{1}\right)^{3} d n+\frac{1}{n_{1}^{4} r_{1}^{4}}(n-1)^{3}\left(r-r_{1}\right)^{4} d n
\end{aligned}
$$


which gives the formula

$$
\begin{aligned}
\mathrm{n}_{1}{ }^{4} Y_{2}= & 4\left(1-n_{1}^{2}\right) P(0,1)+2\left(3-n_{1}^{2}\right) P^{\prime}(0,2)+4 n_{1}^{2} Q^{2}(2) \\
& +4 P^{\prime}(0,3)+2\left(9-n_{1}^{2}\right) P(1,2)-4 n_{1} Q(3)+P^{\prime}(0,4) \\
& +12 P^{\prime}(1,3)+Q(4)+3 P^{\prime}(1,4)+12 P(2,3) \\
& +3 P^{\prime}(2,4)+P(3,4)
\end{aligned}
$$

for the third coefficient in series expansion (4).

It can be shown that, for any positive integer $k$, coefficient $n_{l}{ }^{2 k} Y_{k}$ formed in this way will consist of a sum of $1+2 k(l+k)$ terms, as follows:

i. The terms containing integrals $Q(i)$ are

$$
\begin{gathered}
(-)^{k}\left[\left(2 n_{1}\right)^{k} Q(k)-k\left(2 n_{l}\right)^{k-1} Q(k+1)+\frac{k(k-1)}{2 !}\left(2 n_{1}\right)^{k-2} Q(k+2)\right. \\
\left.-\frac{k(k-1)(k-2)}{3 !}\left(2 n_{l}\right)^{k-3} Q(k+3)+\ldots(-)^{k} Q(2 k)\right] .
\end{gathered}
$$

The total number of these terms is $k+l$.

ii. Each coefficient $\mathrm{n}_{\mathrm{l}}^{2 \mathrm{k}_{\mathrm{Y}}} \mathrm{Y}_{\mathrm{k}}$ contains the odd integrals

$$
\begin{aligned}
k(k-1) & \ldots(k-j+2)(k-j+1)\left[-\frac{2^{j}}{(2 j-1) !}(2 k-1)(2 k-3) \ldots(2 k-2 j+5)(2 k-2 j+3)\right. \\
& -\frac{2^{j-1}}{1 !(2 j-3) !}(2 k-3)(2 k-5) \ldots(2 k-2 j+5)(2 k-2 j+3) n_{1}{ }^{2} \\
& +\frac{2^{j-2}}{2 !(2 j-5) !}(2 k-5)(2 k-7) \ldots(2 k-2 j+5)(2 k-2 j+3) n_{1}{ }^{4} \\
& -\frac{2^{j-3}}{3 !(2 j-7) !}(2 k-7)(2 k-9) \ldots(2 k-2 j+5)(2 k-2 j+3) n_{1} 6 \\
& \left.+\ldots(-)^{j-1} \frac{21}{(j-1) ! 1 !} n_{1}^{2 j-2}\right]\left(1-n_{1}^{2}\right)^{k-2 j+1} P(0,2 j-1)
\end{aligned}
$$

and the even integrals

$$
\begin{aligned}
k(k-1) \ldots(k-j+2)(k-j+1) & {\left[\frac{2 j}{(2 j) !}(2 k-1)(2 k-3) \ldots(2 k-2 j+3)(2 k-2 j+1)\right.} \\
& -\frac{2^{j-1}}{1 !(2 j-2) !}(2 k-3)(2 k-5) \ldots(2 k-2 j+3)(2 k-2 j+1) n_{1}{ }^{2} \\
& +\frac{2^{j-2}}{2 !(2 j-4) !}(2 k-5)(2 k-7) \ldots(2 k-2 j+3)(2 k-2 j+1) n_{1}{ }^{4} \\
& -\frac{2^{j-3}}{3 !(2 j-6) !}-(2 k-7)(2 k-9) \ldots(2 k-2 j+3)(2 k-2 j+1)_{n_{1}} 6 \\
& \left.+\ldots(-)^{j} \frac{2^{0}}{j ! 0 !} n_{1} 2 j\right]\left(1-n_{1}\right)^{k-2 j} P(0,2 j) .
\end{aligned}
$$



terms.

There will be $k$ terms of each kind, or a total of $2 k$

If the multipliers of integrals $P(0,2 j)$ and $P(0,2 j-1)$ are written out as polynomials, in ascending powers of $n_{l}{ }^{2}$, it will be found that their corresponding terms, taken in the order from left to right, have the ratios $(1 / 2 j)(2 k-2 j+l),(1 / 2 j)(2 k-2 j-1), \ldots,(3 / 2 j)$, and $(1 / 2 j)$, respectively. This will be shown by the symbolic notation

$$
P(0,2 j)=\frac{1}{2 j}(2 k-2 j+1,2 k-2 j-1, \ldots, 3,1) P(0,2 j-1) \quad(k \geq j)
$$

the numbers in parentheses indicating term-by-term multiplication of the polynomial coefficient of $\mathrm{P}(0,2 j-1)$ in order to form that of $\mathrm{P}(0,2 j)$. As also holds

$$
P(0,2 j+1)=\left(\frac{2}{2 j+1}\right)(k-j, k-j-1, \ldots, 1,0) P(0,2 j) \quad(k>j)
$$

all the terms given by the direct formulas can be calculated in succession from the first one, $2 \mathrm{k}\left(1-\mathrm{n}_{1}{ }^{2}\right)^{\mathrm{k}-1} \mathrm{P}(0,1)$, the latter developed into a polynomial by the aid of the binomial theorem.

iii. As $\mathrm{y}$ is a symmetric function of $\mathrm{n}$ and $r$, numerous relationships exist that aid the calculation of further terms. Using the symbolic notation, previously introduced, we have

for $k \geq j>i$; a total of $\frac{1}{2} k(k+l)$ terms

$$
P(2 i+1,2 j)=\left(\frac{1}{2 i+1}\right)(2 k-2 i-1,2 k-2 i-3, \ldots, 2 j-2 i+1,2 j-2 i-1) P(2 i, 2 j)
$$

for $k>j>i$, a total of $\frac{1}{2} k(k-1)$ terms

$$
P(2 i+1,2 j+1)=\left(\frac{1}{2 i+1}\right)(2 k-2 i-1,2 k-2 i-3, \ldots, 2 j-2 i+1) P(2 i, 2 j+1)
$$

for $k \geq j>i>0$, a total of $\frac{1}{2} k(k-1)$ terms

$$
P(2 i, 2 j)=\frac{l}{i}(k-i, k-i-1, \ldots, j-i+1, j-i) P(2 i-1,2 j)
$$

and for $k \geq j+l>i>0$, a total of $\frac{1}{2} k(k-1)$ terms

$$
P(2 i, 2 j+1)=\frac{1}{i}(k-i, k-i-1, \ldots, j-i+2, j-i+1) P(2 i-1,2 j+1)
$$

which altogether add $\mathrm{k}(2 \mathrm{k}-1)$ terms to those previously determined.

iv. All the remaining terms are combinative; they will be included simply by priming the integrals $P(i, j)$, excepting those of the form $P(i, i+l)$, which do not possess a counterpart.

We shall not dwell with all the arguments needed in a rigorous proof of these rules by double induction; let it suffice here to show their general validity in a single instance, say, for integral $\mathrm{P}(2,3)$. 
Successive application of the symbolic equations, starting from the last one, gives

$$
P(2,3)=\frac{1}{3}\left[(2 k-1)^{2}(k-1)^{2},(2 k-3)^{2}(k-2)^{2}, \ldots, 9,0\right] P(0,1) .
$$

We know already, from equation (12), that this equality is true if $k=2$. It remains to be shown that if the equality is true for $k=p$, it is also true for $k=p+l$.

To form the polynomial multiplier of $\mathrm{P}(2,3)$ in the coefficient $\mathrm{n}_{1}{ }^{2 \mathrm{p}+2} \mathrm{Y}_{\mathrm{p}+1}$ it is convenient to use a scheme

$$
\begin{array}{ll}
\left.P(2,2)=\frac{1}{2} l(2 p-1)^{2}(p-1),(2 p-3)^{2}(p-2), \ldots, 9,0\right] P(0,1) & \begin{array}{c}
n_{1}{ }^{2}\left(y^{2}-1\right)= \\
\left(2 / r_{1}\right)\left(r-r_{1}\right)
\end{array} \\
P(2,1)=[(2 p-1)(p-1),(2 p-3)(p-2), \ldots, 3,0] P(0,1) & +\left(1 / r_{1}{ }^{2}\right)\left(r-r_{1}\right)^{2} \\
P(1,2)=\frac{1}{2}\left[(2 p-1)^{2},(2 p-3)^{2}, \ldots, 9,1\right] P(0,1) & +\left(4 / r_{1}\right)(n-1)\left(r-r_{1}\right) \\
P(1,1)=(2 p-1,2 p-3, \ldots, 3,1) P(0,1) & +\left(2 / r_{1}{ }^{2}\right)(n-1)\left(r-r_{1}\right)^{2} \\
P(0,2)=\frac{1}{2}(2 p-1,2 p-3, \ldots, 3,1) P(0,1) & +\left(2 / r_{1}\right)(n-1)^{2}\left(r-r_{1}\right) \\
P(0,1)=(1,1, \ldots, 1,1) P(0,1) & +\left(1 / r_{1}{ }^{2}\right)(n-1)^{2}\left(r-r_{1}\right)^{2} \\
P(0,3)=\frac{1}{3}[(2 p-1)(p-1),(2 p-3)(p-2), \ldots, 3,0] P(0,1) & +(n-1)^{2} \\
P(1,3)=\frac{1}{3}\left[(2 p-1)^{2}(1,-1),(2 p-3)^{2}(p-2), \ldots, 9,0\right\} P(0,1) & +2(n-1) \\
P(2,3)=\frac{1}{3}\left[(2 p-1)^{2}(p-1)^{2},(2 p-3)^{2}(p-2)^{2}, \ldots, 100,9,0\right] P(0,1) \quad+\left(1-n_{1}{ }^{2}\right)
\end{array}
$$

which displays the identity for $y^{2}-1$, slightly modified from the form given previously, together with a set of symbolic equations derived by the rules we assume valid. It is now easy to comprehend that the required polynomial is obtained by multiplying each term of the identity into the equation on the left, and adding up the results. These calculations, shown in a separate table, establish the polynomial in the form

$$
P(2,3)=\frac{2}{3} p(p+1)\left[p(2 p+1)^{2},(p-1)(2 p-1)^{2}, \ldots, 50,9\right]\left(1-n_{1}\right)^{p-1}
$$

which is found equivalent to

$$
P(2,3)=\frac{1}{3}\left[p^{2}(2 p+1)^{2},(p-1)^{2}(2 p-1)^{2}, \ldots, 9,0\right](2 p+2)\left(1-n_{1}\right)^{p}
$$

if both formulas are expanded using the binomial theorem. Consequently, the original equality is true for $k=p+l$.

1.3 Calculation of atmospheric integrals $P(i, j)$ and $Q(i)$.

The concept of the atmospheric integrals was introduced in an earlier work (Saastamoinen, 1972-73), where formulas for some of the integrals $P(i, j)$ have been derived on the basis of equations 
Table 1-1. Calculation of the multiplier of integral $P(2,3)$ in $n_{1}{ }^{2 p+2} Y_{p+l}$.
$(2 p-1)^{2}(p-1), \quad(2 p-3)^{2}(p-2), \ldots \ldots \ldots \ldots \ldots, 9, \quad 0$
$(2 p-1)(p-1), \quad(2 p-3)(p-2), \ldots \ldots \ldots \ldots \ldots, 3, \quad 0$
$2(2 \mathrm{p}-1)^{2}, \quad 2(2 \mathrm{p}-3)^{2}, \ldots \ldots \ldots \ldots \ldots \ldots, 18, \quad 2$
$2(2 \mathrm{p}-1), \quad 2(2 \mathrm{p}-3), \ldots \ldots \ldots \ldots \ldots \ldots, 6, \quad 2$
$2 \mathrm{p}-1, \quad 2 \mathrm{p}-3, \ldots \ldots \ldots \ldots \ldots \ldots \ldots, 3, \quad 1$
$1, \quad 1, \ldots \ldots \ldots \ldots \ldots \ldots \ldots \ldots \ldots \ldots \ldots, 1, \quad 1$
$\frac{1}{3}(2 \mathrm{p}-1)(\mathrm{p}-1), \quad \frac{1}{3}(2 \mathrm{p}-3)(\mathrm{p}-2), \ldots \ldots \ldots \ldots \ldots, 1, \quad 0$
$\frac{2}{3}(2 \mathrm{p}-1)^{2}(\mathrm{p}-1), \quad \frac{2}{3}(2 \mathrm{p}-3)^{2}(\mathrm{p}-2), \ldots \ldots \ldots \ldots \ldots, 6, \quad 0$
$\frac{1}{3}(2 \mathrm{p}-1)^{2}(\mathrm{p}-1)^{2}, \frac{1}{3}(2 \mathrm{p}-3)^{2}(\mathrm{p}-2)^{2}, \ldots \ldots \ldots \ldots \ldots, 3, \quad 0$
$0, \quad \frac{1}{3}(2 p-1)^{2}(p-1)^{2}\left(\frac{1}{p-1}\right), \ldots, \frac{100}{3}\left(\frac{p-2}{2 !}\right), \quad 3\left(\frac{p-1}{1}\right)$

Sum: $\left\{\frac{1}{3} p(p+1)(2 p+1)^{2}, \frac{1}{3}(p-1)(p+1)(2 p-1)^{2}, \ldots \ldots, \frac{50}{3}(p+1), 3(n+1)\right\} P(0,1)$

$$
\begin{array}{ll}
n-1=\left(n_{a}-1\right)\left(\frac{T}{T_{a}}\right)^{-\frac{g}{R B}-1} & (\beta \neq 0) \\
n-1=\left(n_{a}-1\right) e^{-\frac{g}{R T}\left(r-r_{a}\right)} & (\beta=0)
\end{array}
$$

in an atmosphere consisting of two laycrs, the troposphere and the stratosphere. We shall no longer restrict the number of layers that may be taken into the atmospheric model, otherwise retaining the various assumptions implicit in equations (13a) and (13b).

In the derivation of a general formula for integrals $F(i, j)$ it is best to take either

$$
\mathrm{H}=\mathrm{RT} / \mathrm{g}
$$

or

$$
h=r-r_{1}
$$

as the independent variable in terms of which all the other quantitie: are expressed. Setting for brevity 


$$
m^{\prime}=-\frac{g}{R \beta}-1
$$

we have then, for $\beta \neq 0$,

$$
\begin{aligned}
& n-1=\left(n_{a}-1\right) H_{a}{ }^{-m^{\prime}} H^{m^{\prime}}=\left(n_{a}-1\right)\left(m^{\prime}+l\right)^{-m^{\prime}} H_{a}{ }^{-m^{\prime}}\left[h_{a}+\left(m^{\prime}+l\right) H a-h\right]^{m^{\prime}} \\
& a n=\left(n_{a}-l\right) m^{\prime} H_{a}^{-m^{\prime}} H^{m^{\prime}-1} d H=-\left(n_{a}-l\right) m^{\prime}\left(m^{\prime}+l\right)^{-m^{\prime}} H_{a}{ }^{-m^{\prime}}\left[h_{a}+\left(m^{\prime}+l\right) H_{a}-h\right]^{m^{\prime}-1} d h \\
& r-r_{I}=h_{a}+\left(m^{\prime}+I\right)\left(H_{a}-H\right)=h_{b}-\left(m^{\prime}+I\right)\left(H-H_{b}\right)=h
\end{aligned}
$$

and

$$
\begin{aligned}
& r_{1}^{j} P(i-l, j)=\left(n_{a}-l\right)^{i} m^{\prime} H_{a}-i m^{\prime} \int_{H_{b}}^{H}\left[h_{a}+\left(m^{\prime}+l\right) H_{a}-\left(m^{\prime}+l\right) H\right]^{j} H^{i m^{\prime}-l} d H \\
& =(i \neq 0) \\
& =\left(n_{a}-1\right)^{i} m^{\prime}\left(m^{\prime}+l\right)^{-i m^{\prime}} H_{a}-i m^{\prime} \int_{h_{a}}^{h}\left[h_{a}+\left(m^{\prime}+l\right) H_{a}-h\right]^{i m^{\prime}-1} h^{j} d h
\end{aligned}
$$

The solution of either integral is given by the formula

$$
\begin{aligned}
& r_{1}^{j} P(i-1, j)=\int_{n_{b}}^{n}(n-1)^{i-1}\left(r-r_{1}\right)^{j} d n \\
& =\frac{j !}{0 ! j^{j}}\left(i m^{\prime}\right)_{j}\left(H_{a}^{j} A_{i}-H_{b}^{j} B_{i}\right)+\frac{j !}{1 ! j^{j-I}}\left(i m^{\prime}\right)_{j-1}\left(H_{a}^{j-1} h_{a} A_{i}-H_{b}^{j-1} h_{b} B_{i}\right) \\
& +\frac{j !}{2 ! i^{j-2}}\left(i m^{\prime}\right) j-2\left(\mathrm{H}^{j-2} h_{a}^{2} A_{i}-H_{b}^{j-2} h_{b}^{2} B_{i}\right) \\
& +\ldots+\frac{j}{i}\left(i m^{\prime}\right)_{1}\left(H_{a} h_{a}^{j-1} A_{i}-H_{b} h_{b}^{j-I_{B}}\right)+h_{a}{ }^{j} A_{i}-h_{b}{ }^{j} B_{i}
\end{aligned}
$$

where

$$
A_{i}=\frac{1}{i}\left(n_{a}-1\right)^{i} \quad B_{i}=\frac{1}{i}\left(n_{b}-1\right)^{i}
$$

and

$\frac{i m^{\prime}+i}{i m^{\prime}+1}=\left(i m^{\prime}\right)_{1},\left(\frac{i m^{\prime}+i}{i m^{\prime}+2}\right)\left(i m^{\prime}\right)_{1}=\left(i m^{\prime}\right)_{2}, \ldots,\left(\frac{i m^{\prime}+i}{i m^{\prime}+j}\right)\left(i m^{\prime}\right)_{j-1}=\left(i m^{\prime}\right)_{j}$.

Because

$\lim _{\beta \rightarrow 0}\left(\operatorname{in}^{\prime}\right)_{j}=1$ 
it is evident that equation (14) is also valid if $\beta=0$, in which case the factors containing $\mathrm{m}^{\prime}$ are simply left out.

For integrals $Q(i)$, the immediate solution is

$$
\begin{aligned}
Q(i) & =\int_{n_{b}}^{n} \frac{1}{n}\left(n_{1}-n\right)^{i} d n \\
= & n_{1}{ }^{i} \log _{e}\left(n_{a} / n_{b}\right)-i n_{1}{ }^{-1}\left(n_{a}-n_{b}\right)+\frac{1}{2} \frac{i(i-1)}{2 !} n_{l}{ }^{i-2}\left(n_{a}{ }^{2}-n_{b}{ }^{2}\right) \\
& -\frac{1}{3} \frac{i(i-1)(i-2)}{3 !} n_{1}^{i-3}\left(n_{a}{ }^{3}-n_{b}{ }^{3}\right)+\ldots(-)^{i} \frac{1}{i}\left(n_{a}{ }^{i}-n_{b}{ }^{i}\right) .
\end{aligned}
$$

This expression may be transformed, setting

$$
A=n_{a}-n_{b} \quad c=\frac{1}{2}\left(n_{a}+n_{b}\right)
$$

and

$$
\log _{e}\left(n_{a} / n_{b}\right)=(A / C)+\frac{1}{2^{2} \cdot 3}(A / C)^{3}+\frac{1}{2^{4} \cdot 5}(A / C)^{5}+\ldots,
$$

into the following formula suitable for numerical evaluation,

$$
\begin{aligned}
& Q(i)=\left(n_{1}-C\right)^{i}(A / C) \\
& +\frac{1}{2^{2} \cdot 3}\left[n_{1}{ }^{2}+(i-2) n_{1} C+\frac{1}{2 !}(i-1)(i-2) C^{2}\right]\left(n_{1}-C\right)^{i-2}(A / C)^{3} \\
& +\frac{1}{2^{4} \cdot 5}\left[n_{1}{ }^{4}+(i-4) n_{1}{ }^{3} C+\frac{1}{2 !}(i-3)(i-4) n_{1}{ }^{2} C^{2}+\frac{1}{3 !}(i-2)(i-3)(i-4) r_{1} C^{3}\right. \\
& \left.\quad+\frac{1}{4 !}(i-1)(i-2)(i-3)(i-4) C^{4}\right]\left(n_{1}-C\right)^{i-4}(A / C)^{5} \\
& +\frac{1}{2^{6} \cdot 7}\left[n_{1}{ }^{6}+(i-6) n_{1}{ }^{5} C+\ldots+\frac{1}{6 !}(i-1)(i-2) \ldots(i-6) C^{6}\right]\left(n_{1}-C\right)^{i-6}(A / C)^{7} \\
& +\ldots,
\end{aligned}
$$

where $i$ may take any value $(i=0,1,2, \ldots)$.

1.4 Extension of the range of the refraction formula.

We shall now go back to the binomial expansion for $\tan \mathrm{z}$, and replace equation (3) by

$$
\begin{aligned}
& \tan z=f \tan z_{1}\left[1+f^{2}\left(y^{2}-1\right) \sec ^{2} z_{1}-\left(1-f^{2}\right)\right]^{-\frac{1}{2}} \\
& =f \tan z_{1}\left\{1+(-)^{k} \sum_{k=1}^{\infty} \frac{1 \cdot 3 \cdot 5 \ldots(2 k-1)}{2 \cdot 4 \cdot 6 \ldots(2 k)}\left[f^{2}\left(y^{2}-1\right) \sec ^{2} z_{1}-\left(1-f^{2}\right)\right]^{k}\right\}
\end{aligned}
$$

where $f$ stands for a positive number less than 1 . This series is convergent if $\left|f^{2}\left(y^{2}-1\right) \sec ^{2} z-\left(1-f^{2}\right)\right|<I$; consequently, it can be made convergent for any zenith distance $z_{1}<90^{\circ}$ by taking $f$ sufficiently small. 
The idea behind this arrangement is to truncate series (16), after $k+1$ terms, whereupon it is permissible to restore the original order of terms in ascending powers of $\mathrm{y}^{2}-1$. The contribution to the astronomical refraction of a layer of air between radii vectors $r_{a}$ and $r_{b}$ then becomes

$$
\begin{aligned}
\Delta z_{1}(a ; b)= & \tan z_{1}\left[F_{0} Y_{0}-\frac{1}{2} F_{1} Y_{1} \sec ^{2} z_{1}+\frac{3}{8} F_{2} Y_{2} \sec ^{4} z_{1}-\frac{5}{16} F_{3} Y_{3} \sec ^{6} z_{1}\right. \\
& \left.+\ldots(-)^{k} \frac{1 \cdot 3 \ldots(2 k-1)}{2 \cdot 4 \ldots(2 k)} F_{k} Y_{k} \sec ^{2 k} z_{1}\right]+R_{k+1}
\end{aligned}
$$

where

$$
\begin{aligned}
& F_{0}=f\left\{1+\frac{1}{2}\left(1-f^{2}\right)+\ldots+\frac{1 \cdot 3 \ldots(2 k-1)}{2 \cdot 4 \ldots(2 k)}\left(1-f^{2}\right)^{k}\right\} \\
& F_{1}=f^{3}\left\{1+\frac{3}{2}\left(1-f^{2}\right)+\ldots+\frac{3 \cdot 5 \ldots(2 k-1)}{2 \cdot 4 \ldots(2 k-2)}\left(1-f^{2}\right)^{k-1}\right\} \\
& F_{2}=f^{5}\left\{1+\frac{5}{2}\left(1-f^{2}\right)+\ldots+\frac{5 \cdot 7 \ldots(2 k-1)}{2 \cdot 4 \ldots(2 k-4)}\left(1-f^{2}\right)^{k-2}\right\} \\
& \ldots \ldots \ldots, \\
& F_{k-I}=f^{2 k-1}\left\{1+\frac{2 k-1}{2}\left(1-f^{2}\right)\right\} \\
& F_{k}=f^{2 k+1} .
\end{aligned}
$$

Formula (17) is valid if

$$
\mathrm{f}^{2}<\frac{2}{\left(\mathrm{y}_{\mathrm{b}}{ }^{2}-1\right) \sec ^{2} \mathrm{z}_{1}+1}
$$

but in order to keep remainder $R_{k+l}$ smail, without the necessity of including an excessive number of terms, $f$ should be chosen so that

$$
\left|f^{2}-\frac{1}{\left(y^{2}-1\right) \sec ^{2} z_{1}+1}\right|=\min \text {. }
$$

considering all the values of $\mathrm{y}$ and $\mathrm{z}_{1}$ involved.

With given numerical values of $\mathrm{f}$ and $\mathrm{k}$, equations (18) provide a set of damping factors $, F_{0}, F_{1}, \ldots, F_{k}$, by which the coefficients of the first $\mathrm{k}+\mathrm{l}$ terms of refraction formula (4) are multiplied.

Upon truncation of series (16), the remaining terms of tan $z$ are

$$
\begin{gathered}
(-)^{k+1} \frac{1 \cdot 3 \cdot 5 \ldots(2 k+1)}{2 \cdot 4 \cdot 6 \ldots(2 k+2)} f \tan z_{1}\left[I-\left(\frac{2 k+3}{2 k+4}\right) v+\frac{(2 k+3)(2 k+5)}{(2 k+4)(2 k+6)} v^{2}\right. \\
\left.-\frac{(2 k+3)(2 k+5)(2 k+7)}{(2 k+4)(2 k+6)(2 k+8)} v^{3}+\ldots\right] v^{k+1}
\end{gathered}
$$

where

$$
V=f^{2}\left(y^{2}-1\right) \sec ^{2} z-\left(1-f^{2}\right)
$$

This gives the approximate formuia 


$$
R_{k+1} \sim(-)^{k+1} \frac{1 \cdot 3 \cdot 5 \ldots(2 k+1)}{2 \cdot 4 \cdot 6 \ldots(2 k+2)} f \tan z_{l} \int_{n_{b}}^{n} \frac{v^{k+1}}{1+\frac{2 k+3}{2 k+4} v} d n
$$

with

$$
\mathrm{dn}=-\left(\frac{m^{\prime}}{m^{\prime}+1}\right)\left(\frac{n-1}{H}\right) d r
$$

for the evaluation of the magnitude of remainder $\mathrm{R}_{\mathrm{k}+1}$ by numerical integration. This evaluation is necessary because the $(k+l)$ th term of formula (17), unlike that of equation (4), does not give an indication of the accuracy achieved.

If tan $z_{1}$ is numerically large, we may choose to substitute $f$ cos $z_{l}$ for $f$ in equation (16), and consider the series

$$
\begin{aligned}
& \tan z^{\prime}=f \sin z_{1}\left[1+f^{2}\left(y^{2}-1\right)-\left(1-f^{2} \cos ^{2} z_{1}\right)\right]^{-\frac{1}{2}} \\
& =f \sin z_{1}\left\{1+(-)^{k} \sum_{k=1}^{\infty} \frac{1 \cdot 3 \cdot 5 \ldots(2 k-1)}{2 \cdot 4 \cdot 6 \ldots(2 k)}\left[f^{2}\left(y^{2}-1\right)-\left(1-f^{2} \cos ^{2} z_{1}\right)\right]^{k}\right\}
\end{aligned}
$$

where $f$ now stands for a positive number greater than 1 . The contribution to the astronomical refraction of a layer of air between radii vectors $r_{a}$ and $r_{b}$ then becomes

$$
\begin{aligned}
\Delta \mathrm{z}_{1}(\mathrm{a} ; \mathrm{b})= & \sin \mathrm{z}_{1}\left[\mathrm{~F}_{0} \mathrm{Y}_{0}-\frac{1}{2} \mathrm{~F}_{1} \mathrm{Y}_{1}+\frac{3}{8} \mathrm{~F}_{2} \mathrm{Y}_{2}-\frac{5}{16} \mathrm{~F}_{3} \mathrm{Y}_{3}\right. \\
& \left.+\ldots(-)^{\mathrm{k}} \frac{1 \cdot 3 \cdot 5 \ldots(2 \mathrm{k}-1)}{2 \cdot 4 \cdot 6 \ldots(2 \mathrm{k})} \mathrm{F}_{\mathrm{k}} \mathrm{Y}_{\mathrm{k}}\right]+\mathrm{R}_{\mathrm{k}+1}
\end{aligned}
$$

where the damping factors

$$
\begin{aligned}
& F_{0}=f\left\{1+\frac{1}{2}\left(1-f^{2} \cos ^{2} z_{1}\right)+\ldots+\frac{1 \cdot 3 \ldots(2 k-1)}{2 \cdot 4 \ldots(2 k)}\left(1-f^{2} \cos ^{2} z_{1}\right)^{k}\right\} \\
& F_{1}=f^{3}\left\{1+\frac{3}{2}\left(1-f^{2} \cos ^{2} z_{1}\right)+\ldots+\frac{3 \cdot 5 \ldots(2 k-1)}{2 \cdot 4 \ldots(2 k-2)}\left(1-f^{2} \cos ^{2} z_{1}\right)^{k-1}\right\} \\
& F_{2}=f^{5}\left\{1+\frac{5}{2}\left(1-f^{2} \cos ^{2} z_{1}\right)+\ldots+\frac{5 \cdot 7 \ldots(2 k-1)}{2 \cdot 4 \ldots(2 k-4)}\left(1-f^{2} \cos ^{2} z_{1}\right)^{k-2}\right\} \\
& \ldots \ldots \\
& F_{k-1}=f^{2 k-1}\left\{1+\frac{2 k-1}{2}\left(1-f^{2} \cos ^{2} z_{1}\right)\right\} \\
& F_{k}=f^{2 k+1}
\end{aligned}
$$

may be written out as polynomials of $\cos ^{2} z_{1}$. Series (16') is convergent if

$$
f^{2}<\frac{2}{y_{b}^{2}-\sin ^{2} z_{1}}
$$


but again, f should be chosen so that

$$
\left|\mathrm{t}^{2}-\frac{1}{\mathrm{y}^{2}-\sin ^{2} z_{1}}\right|=\min
$$

for all the values of $\mathrm{y}$ and $\mathrm{z}_{\mathrm{l}}$ involved. Remainder $\mathrm{R}_{\mathrm{k}+\mathrm{l}}$ is evaluated by numerical integration using the formulas

$$
\begin{aligned}
& W=f^{2}\left(y^{2}-1\right)-\left(1-f^{2} \cos ^{2} z_{I}\right) \\
& a n=-\left(\frac{m^{\prime}}{m^{\prime}+1}\right)\left(\frac{n-1}{H}\right) d r
\end{aligned}
$$

and

$$
R_{k+1} \sim(-)^{k+1} \frac{1 \cdot 3 \cdot 5 \ldots(2 k+1)}{2 \cdot 4 \cdot 6 \ldots(2 k+2)}+\sin z_{1} \int_{n_{b}}^{n} \frac{w^{k+1}}{1+\frac{2 k+3}{2 k+4} w} d n .
$$

If $z_{1}=90^{\circ}$, equation (17) can not be applied unless the point of observation is moved along the light ray to the base $\left(r_{a}\right)$ of a higher layer, where

$$
\tan z_{a}=\left\{\left[n_{a} r_{a} /\left(n_{1} r_{1} \sin z_{1}\right)\right]^{2}-1\right\}^{-\frac{1}{2}}
$$

has a suitable value. The refraction component for the nearly horizontal section of the light ray may be calculated using the series expansions in 1.5 .

1.5 Calculation of astronomical refraction at or near the horizon.

We shall now consider the integral

$$
\Delta z_{l}(a ; b)=\sin z_{a} \int_{\log _{e} n_{b}}^{\log _{e} n a}\left(n^{2}-\sin ^{2} z_{a}\right)^{-\frac{1}{2}} \operatorname{dlog} n
$$

where

$$
n=n r /\left(n_{a} r_{a}\right)
$$

in the calculation of astronomical refraction for any zenith distance $0^{\circ} \leq z_{a} \leq 90^{\circ}$, especially for $z_{a}=90^{\circ}$. In the solution given below, the integrand will be expressed in the form of a convergent series, and integrated term by term.

Let

$$
x=\left(r-r_{a}\right) / H_{a}
$$


be chosen as the independent variable. We have then, from equation (13a),

$$
\begin{aligned}
& \left(\mathrm{n}_{1}-1\right) /\left(\mathrm{n}_{\mathrm{a}}-1\right)=\left(1-\frac{\mathrm{x}}{\mathrm{m}^{\prime}+1}\right)^{m^{\prime}} \\
& \quad=1-\mathrm{m}_{1} \mathrm{x}+\mathrm{m}_{1} \mathrm{~m}_{2} \mathrm{x}^{2}-\mathrm{m}_{1} \mathrm{~m}_{2} \mathrm{~m}_{3} \mathrm{x}^{3}+\ldots
\end{aligned}
$$

and

$$
n / n_{a}=I-a_{0} m_{1} x+a_{0} m_{1} m_{2} x^{2}-a_{0} m_{1} m_{2} m_{3} x^{3}+\ldots
$$

with

$$
a_{0}=\left(n_{a}-1\right) / n_{a} ; \quad m_{j}=\frac{1}{j}-\frac{1}{m^{\prime}+1} \quad\left(\text { or } m_{j}=\frac{1}{j} \text {, if } \beta=0\right) \text {. }
$$

The derivative of series (22)

$$
\frac{d n}{d x} / n_{a}=-a_{0} m_{1}+2 a_{0} m_{1} m_{2} x-3 a_{0} m_{1} m_{2} m_{3} x^{2}+\ldots
$$

divided by the series itself gives

$$
\begin{aligned}
& \operatorname{dlog}_{e} n=-a_{0} m_{1}\left[1-\left(2 m_{2}-a_{0} m_{1}\right) x+\left(3 m_{2} m_{3}-3 a_{0} m_{1} m_{2}+a_{0}{ }^{2} m_{1}{ }^{2}\right) x^{2}\right. \\
& \left.-\left(4 m_{2} m_{3} m_{4}-4 a_{0} m_{1} m_{2} m_{3}-2 a_{0} m_{1} m_{2}^{2}+4 a_{0}{ }^{2} m_{1}{ }^{2} m_{2}-a_{0}{ }^{3} m_{1}{ }^{3}\right) x^{3}+\ldots\right] d x
\end{aligned}
$$

Multiplication of series (22) by the binom

$$
r / r_{a}=I+\left(H_{a} / r_{a}\right) x=I+b_{0} x
$$

further gives

$$
n=1+\left(b_{0}-a_{0} m_{1}\right) x+a_{0} m_{1}\left(m_{2}-b_{0}\right) x^{2}-a_{0} m_{1} m_{2}\left(m_{3}-b_{0}\right) x^{3}+\ldots
$$

whence

$$
\begin{aligned}
& n^{2}-\sin ^{2} z_{a}=\cos ^{2} z_{a}+2\left(b_{0}-a_{0} m_{1}\right) x+2\left(a_{0} m_{1} m_{2}-2 a_{0} m_{1} b_{0}\right. \\
+ & \left.\frac{1}{2} a_{0}^{2} m_{1}^{2}+\frac{1}{2} b_{0}^{2}\right) x^{2}-2 a_{0} m_{1}\left(m_{2} m_{3}-2 b_{0} m_{2}+a_{0} m_{1} m_{2}-a_{0} m_{1} b_{0}+b_{0}{ }^{2}\right) x^{3}+\ldots \\
& =\cos ^{2} z_{a}+c_{1} x+c_{2} x^{2}-c_{3} x^{3}+\ldots
\end{aligned}
$$

The remaining part of the calculation consists of the extraction of the inverse square root of series (24), multiplication by differential (23), and integration of the product according to equation (21).

$$
\begin{aligned}
& \text { If } z_{a}=90^{\circ}, \text { we have } \sin ^{2} z_{a}=1, \cos ^{2} z_{a}=0 \\
& \eta^{2}-1=c_{1} x\left(1+\frac{c_{2}}{c_{1}} x-\frac{c_{3}}{c_{1}} x^{2}+\ldots\right)=c_{1} x(1+x) \\
& \left(\eta^{2}-1\right)^{-\frac{1}{2}}=\frac{1}{\sqrt{c_{1}}} x^{-\frac{1}{2}}\left(1-\frac{1}{2} x+\frac{3}{8} x^{2}-\frac{5}{16} x^{3}+\ldots\right)
\end{aligned}
$$


The integrand is obtained in the form

$$
\left(n^{2}-1\right)^{-\frac{1}{2}} \operatorname{llog}_{e} n=-\frac{a_{0} m_{1}}{\sqrt{c_{1}}} x^{-\frac{1}{2}}\left(1-a_{1} x+d_{2} x^{2}-\ldots\right) d x
$$

with

$$
\begin{aligned}
& a_{1}=2 m_{2}-a_{0} m_{1}+\frac{1}{2}\left(c_{2} / c_{1}\right) \\
& d_{2}=3 m_{2}\left(m_{3}-a_{0} m_{1}\right)+\frac{1}{2 c_{1}}\left(2 c_{2} m_{2}+c_{3}-a_{0} c_{2} m_{1}\right)+\frac{3}{8}\left(c_{2} / c_{1}\right)^{2}+a_{0}{ }^{2} m_{1}{ }^{2} \\
& \ldots \ldots \ldots
\end{aligned}
$$

which gives

$$
\Delta \mathrm{z}_{1}(\mathrm{a} ; \mathrm{b})=\frac{2 \mathrm{a}_{0} \mathrm{~m}_{1}}{\sqrt{\mathrm{c}_{1}}} \sqrt{\mathrm{x}_{\mathrm{b}}}\left(1-\frac{1}{3} \mathrm{a}_{1} \mathrm{x}_{\mathrm{b}}+\frac{1}{5} \mathrm{a}_{2} \mathrm{x}_{\mathrm{b}}{ }^{2}-\ldots\right)
$$

as the final result.

If $\cos ^{2} z$ is numerically small, the extraction of the inverse square root succeeds similarly if we first find (by iteration) a number, $\mathrm{x}_{0}$, such that

$$
\cos ^{2} z_{a}-c_{1} x_{0}+c_{2} x_{0}^{2}+c_{3} x_{0}^{3}+\ldots=0
$$

and substitute a new variable $\mathrm{w}=\mathrm{x}+\mathrm{x}_{0}$ that eliminates the constant term in equation (24). This procedure, which is equivalent to extending the light ray to its lowest point where the tangent line is horizontal, also finds application in the calculation of refraction if $z_{a}>90^{\circ}$.

$$
\text { (to be continued) }
$$

\section{REFERENCES}

Saastamoinen, J.: 1972-73, "Corttributions to the theory of atmospheric refraction", Bulletin Géodésique, Nos. 105-107

Teleki, G.: (edited by) "The present state and future of the astronomical refraction investigations". Proceedings of the Study Group on Astronomical Refraction of the International Astronomical Union Commission 8, Belgrade, 1974.

APPENDIX A

Numerical applications

A few illustrative samples are given on the calculation of astronomical refraction in a spherically symmetric model (Atmospheric Hodel No. 2, (Saastamoinen, 1972-73)) specifica as follows: 


$$
r_{l}=6380 \mathrm{~km} \quad \mathrm{~T}_{1}=285.08 \mathrm{~K} \quad \mathrm{n}_{1}=1.000280868
$$

Troposphere $0-10.4 \mathrm{~km}: \quad \beta=-6.45 \mathrm{~K} \cdot \mathrm{km}^{-1} \mathrm{R} / \mathrm{g}=(2.8704 / 98) \mathrm{km} \cdot \mathrm{K}^{-1}$ Stratosphere $10.4-\infty \mathrm{km}: \quad B=0 \quad \mathrm{R} / \mathrm{g}=(2.8704 / 98) \mathrm{km} \cdot \mathrm{K}^{-1}$

The diminution of the refractive index with height is given by equations (13a) and (13b); for the purpose of calculation, the specified values are assumed to be exact.

1. Coefficients $\mathrm{Y}_{\mathrm{k}}$.

\begin{tabular}{|c|c|c|c|c|}
\hline Layer: & $0-10.1 \mathrm{~km}$ & $10.4-24 \mathrm{~km}$ & $2 h-\infty \mathrm{km}$ & $\begin{array}{l}\text { Binomia? } \\
\text { multiplier }\end{array}$ \\
\hline$Y_{0}$ & $39: 614630$ & $16 " 134229$ & $2 " 176190$ & 1 \\
\hline $10^{2} Y_{1}$ & 4.768940 & 6.821834 & 1.956960 & $-1 / 2$ \\
\hline $10^{4} \mathrm{Y}_{2}$ & 0.834121 & 3.067383 & 1.847449 & $3 / 8$ \\
\hline $10^{6} \mathrm{Y}_{3}$ & 0.170238 & 1.466399 & 1.854364 & $-5 / 16$ \\
\hline $10^{8} \mathrm{Y}_{4}$ & 0.037758 & 0.742198 & 2.007800 & $35 / 128$ \\
\hline $10^{10} \mathrm{Y}_{5}$ & 0.008818 & 0.394937 & 2.379461 & $-63 / 256$ \\
\hline $10^{12} y_{6}$ & 0.002132 & 0.219156 & 3.124625 & $231 / 102 / 4$ \\
\hline $10^{14} Y_{7}$ & 0.000529 & 0.125831 & 4.581027 & $-429 / 2048$ \\
\hline $10^{16} Y_{8}$ & 0.000133 & 0.074246 & 7.511767 & $6435 / 32768$ \\
\hline $10^{18} \mathrm{Y}_{9}$ & 0.000034 & $0.04477 ?$ & $13.73 ? 74.43$ & $-12155 / 65536$ \\
\hline
\end{tabular}

2. Examples of formulas for astronomical refraction.

$s=10^{-2} \sec ^{2} z_{1}$

Zenith distances $0^{\circ} \leq z_{1} \leq 80^{\circ}$.

$$
\begin{aligned}
\Delta z_{1}=\tan & z_{1}\left(57.992505-6.77387 s+2.15586 s^{2}-1.09094 s^{3}\right. \\
+ & 0.176228 s^{4}-0.68493 s^{5}+0.75479 s^{6}-0.98607 s^{7} \\
+ & \left.1.48977 s^{8}-2.55514 s^{9}\right) \cdot \quad \max R_{10} \sim 0.0003
\end{aligned}
$$




$$
\begin{aligned}
& \text { Zenith distances } 0^{\circ} \leq z_{1} \leq 82^{\circ} \text {. } \\
& (f=0.9) \\
& \Delta z_{1}=\tan z_{1}\left(57.92505-6.77386 s+2.15580 s^{2}-1.09052 s^{3}\right. \\
& +0.75966 s^{4}-0.67073 s^{5}+0.68851 s^{6}-0.72595 s^{7} \\
& \left.+0.64970 s^{8}-0.134516 s^{9}\right) \text {. } \\
& \max R_{10} \sim 0.0001 \\
& \text { Zenith aistances } 82^{\circ} \leq z_{1} \leq 84^{\circ} \text {. } \\
& (\mathrm{f}=0.9,0-10.4 \mathrm{~km} \text {; } \\
& f=0.75,10.4-\infty \mathrm{km}) \\
& \Delta z_{1}=\tan z_{1}\left(57 " .92398-6 " .76680 s+2 " 13081 s^{2}-1.02570 s^{3}\right. \\
& +0.61 .974 s^{4}-0.040724 s^{5}+0.25657 s^{6}-0.13688 s^{7} \\
& \left.+0.05285 s^{8}-0.01080 s^{9}\right) \text {. } \\
& \max R_{10} \sim 0 . " 0001 \\
& (f=0.9,0-10.4 \mathrm{~km} \text {; } \\
& \left.f^{\prime}=0.75,10.4-24 \mathrm{~km} ; f=0.56,24-\infty \mathrm{km}\right) \\
& \Delta z_{1}=\tan z_{1}\left(57.909480-6.696487 s+1 . " 962573 s^{2}-0.1758515 s^{3}\right. \\
& +0.3019568 s^{4}-0.1082585 s^{5}+0.03197350 s^{6}-0.007131299 s^{7} \\
& \left.+0.001056695 s^{8}-0.000077801 s^{9}\right) . \quad \max R_{10} \sim 0 " .001
\end{aligned}
$$


3. Contributions of the atmospheric integrals to the coefficients

$\begin{array}{lllll}\begin{array}{l}\text { Inte- } \\ \text { gral }\end{array} & \mathrm{k}=0 & \mathrm{k}=1 & \mathrm{k}=2 & \mathrm{k}=3\end{array}$

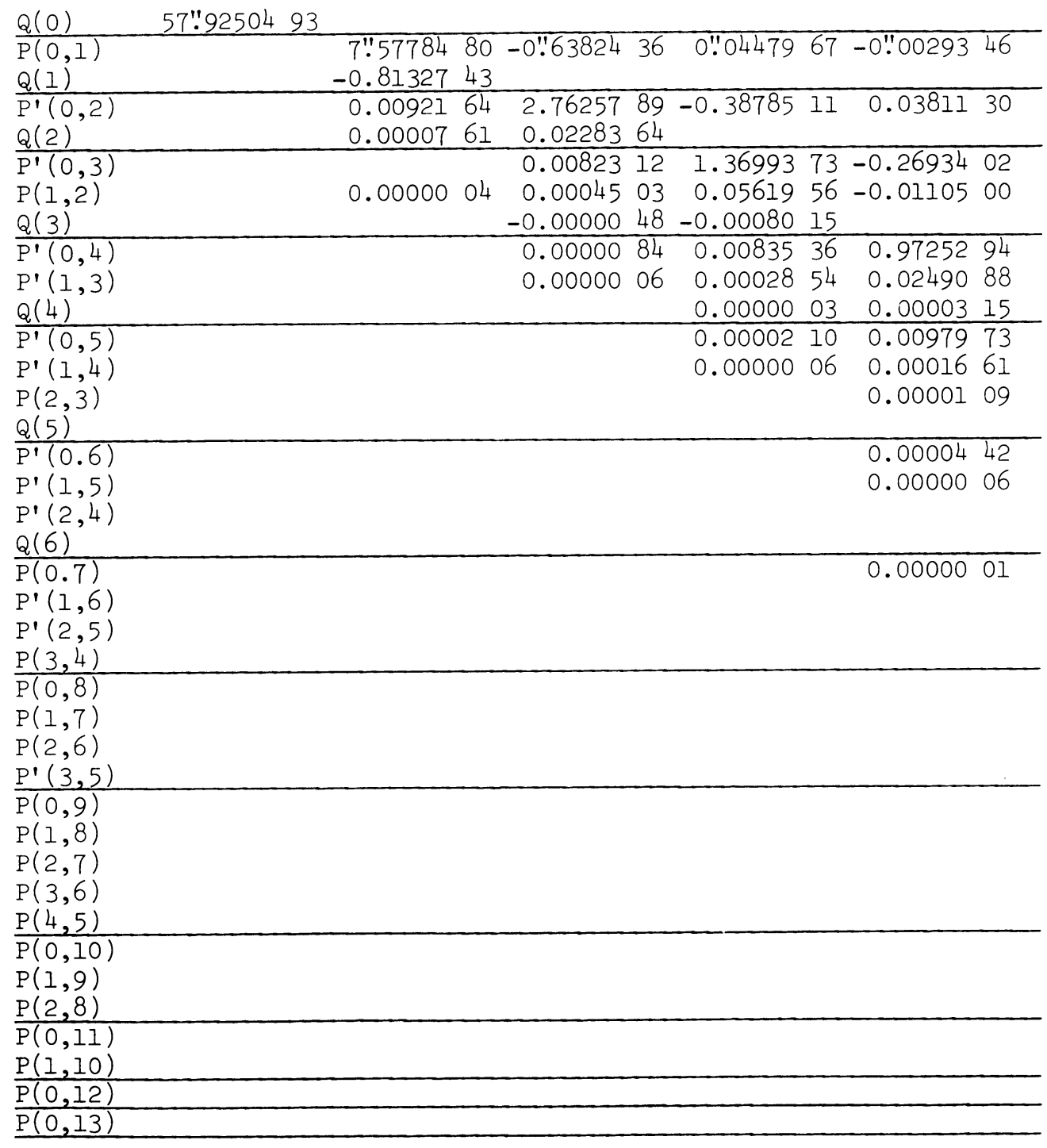

Totals 57".92504 93 6".77386 66 2".15585 74 1"09093 79 0".76227 71 
in the formula for astronomical refraction.

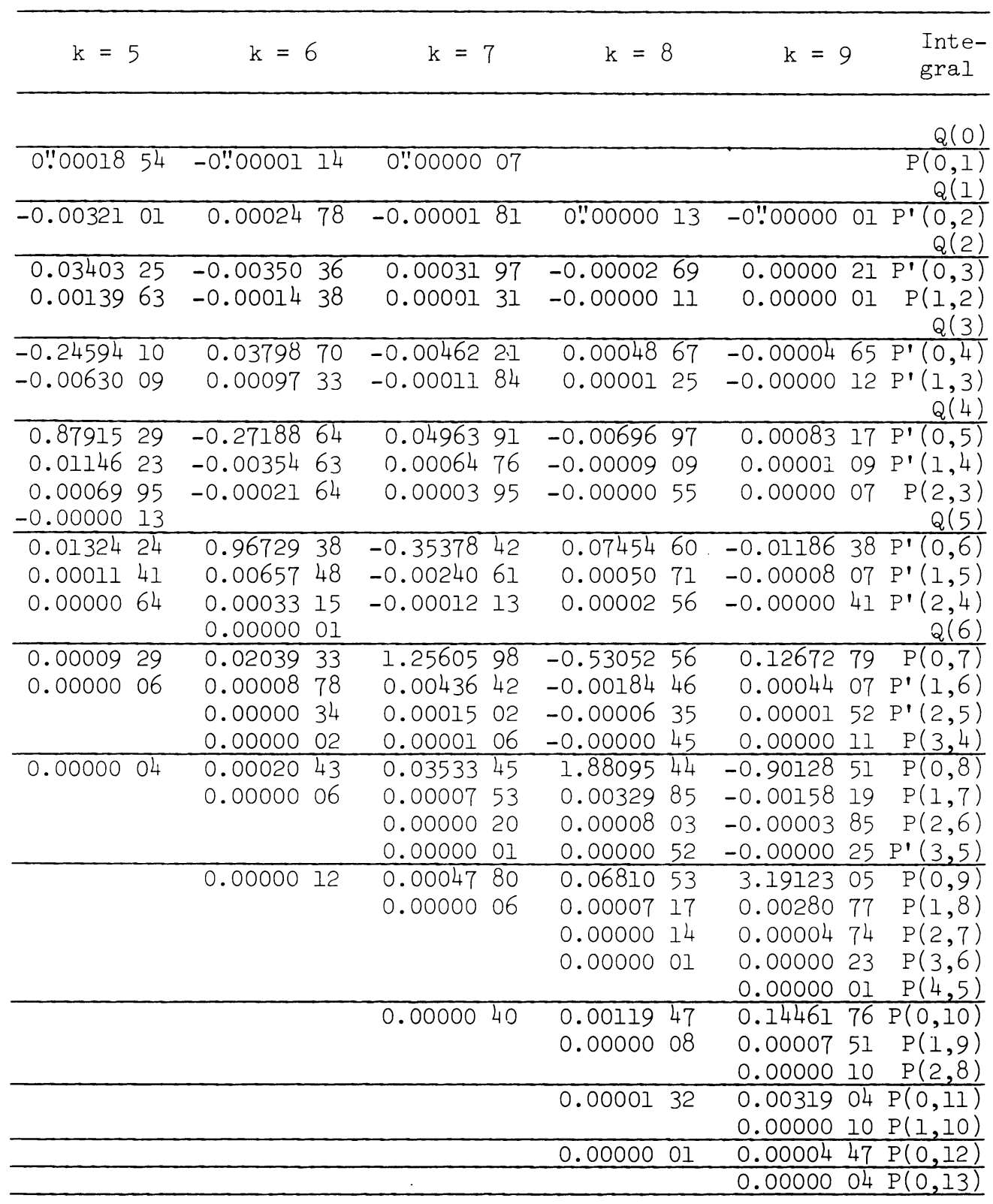

0.68493 $24 \quad$ 0."75479 $12 \quad$ 0.98606 $88 \quad$ 1."48977 $26 \quad$ 2".55514 42 Totals 
4. Sample calculation of $\mathrm{R}_{\mathrm{k}+1}$.

$\left(\mathrm{k}=9 ; \mathrm{z}_{1}=85^{\circ}\right)$

$\mathrm{h}, \mathrm{km} \quad-1.0^{6} \frac{\mathrm{dn}}{\mathrm{dr}} \quad 10^{2}\left(\mathrm{y}^{2}-\mathrm{l}\right) \quad \mathrm{v} \quad-0.4154\left(\frac{\mathrm{dn}}{\mathrm{dr}}\right) \mathrm{f} \mathrm{V}^{10} /\left(1+\frac{2 \mathrm{l}}{22} \mathrm{~V}\right)$

\begin{tabular}{rrrrrr}
\hline 0 & 27.28 & 0.0000 & -0.1900 & $0.164 \times 10^{-6}$ \\
2.6 & 22.34 & 0.0686 & -0.1168 & 0.004 \\
5.2 & 18.07 & 0.1397 & -0.0410 & 0.000 \\
7.8 & 14.39 & 0.2128 & 0.0369 & 0.000 & $f=0.9$ \\
10.4 & 11.28 & 0.2878 & 0.1168 & 0.002 & \\
\hline 10.4 & 13.90 & 0.2878 & -0.2244 & 1.786 \\
13.8 & 8.163 & 0.3871 & -0.1508 & 0.018 \\
17.2 & 4.793 & 0.4896 & -0.0749 & 0.000 \\
20.6 & 2.814 & 0.5939 & 0.0023 & 0.000 \\
24 & 1.652 & 0.6993 & 0.0804 & 0.000 & $f=0.75$ \\
\hline 24 & 1.6523 & 0.6993 & -0.3977 & 61.31 & \\
36 & 0.2523 & 1.075 & -0.242 & 0.05 & \\
48 & 0.0385 & 1.453 & -0.086 & 0.00 & $f=0.56$ \\
60 & 0.0059 & 1.833 & 0.070 & 0.00 & \\
72 & 0.0009 & 2.212 & 0.227 & 0.00 & \\
\hline
\end{tabular}

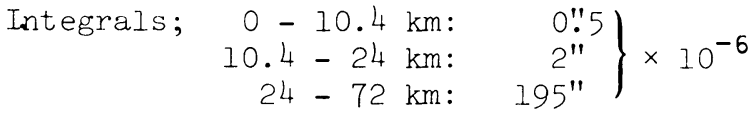

$R_{10} \sim 0.0002$

5. Astronomical refraction in the troposphere at $z_{1}=90^{\circ}$.

$$
\mathrm{x}=0.1197614110 \mathrm{~h} \quad(\mathrm{~h} \text { in } \mathrm{km})
$$

$\Delta z_{1}\left(r_{1} ; r\right)=2020.53687 \sqrt{x}\left(1-0.21827814 x+0.032260119 x^{2}\right.$

$-0.002864275 x^{3}+0.000217379 x^{4}-0.000023471 x^{5}+0.000002731 x^{6}$

$\left.-0.000000321 x^{7}+0.000000040 x^{8}-0.000000005 x^{9}\right)$.

(Total 1743".330). 
APPENDIX B

Coefficients $\mathrm{Y}_{\mathrm{K}}$

1. Polynomial multipliers of integrals $P(i, j)$ in $n_{l}{ }^{2 k_{Y}}{ }_{k}$.

$k(k-1) \ldots(k-p+l)(k-p)\left(P_{n}(i, j)\left(1-n_{1}^{2}\right)^{k-i-j} P(i, j)\right.$

where

$$
\begin{aligned}
& p=\frac{1}{2}(j-2) \text { if } j \text { is even, or } \\
& p=\frac{1}{2}(j-1) \text { if } j \text { is oda. } \\
& P_{n}(0,1)=2 \\
& P_{n}(0,2)=(2 k-1)-n_{1}^{2} \\
& \frac{3}{2} P_{n}(0,3)=(2 k-1)-3 n 1^{2} \\
& P_{n}(1,2)=(2 k-1)^{2}-(8 k-6) n_{1}{ }^{2}+n_{1}^{4} \\
& 6 \mathrm{P}_{\mathrm{n}}(0,4)=(2 \mathrm{k}-1)(2 \mathrm{k}-3)-6(2 \mathrm{k}-3) \mathrm{n}_{1}^{2}+3 \mathrm{n}^{4} \\
& \frac{3}{2} P_{n}(1,3)=(2 k-1)^{2}-2(8 k-7) n l^{2}+9 n 1^{4} \\
& 15 P_{n}(0,5)=(2 k-1)(2 k-3)-5(4 k-6) n_{1}^{2}+15 n_{1}^{4} \\
& 6 y_{n}(i, l ;)=(2 k-1)^{2}(2 k-3)-(2 k-3)(26 k-25) n_{1}{ }^{2}+3(22 k-35) n_{1}{ }^{4}-9 n_{1}^{6} \\
& \frac{3}{2} n(2,3)=(k-1)(2 k-1)^{2}-\left(28 k^{2}-53 k+3\right) n_{1}{ }^{2}+(4+k-55) n_{1}{ }^{4}-9 n_{1} 6 \\
& E n_{n}(0,6)=\frac{1}{15}(2 k-1)(2 k-3)(2 k-5)-(2 k-3)(2 k-5) n_{1}^{2}+3(2 k-5) n_{1}{ }^{4}-n_{1}{ }^{6} \\
& 15 F_{n}(i, 5)=(2 k-1)^{2}(2 k-3)-(2 k-3)(33 k-39) n_{1}^{2}+5(34 k-57) n_{1}{ }^{4}-75 n_{1}{ }^{6} \\
& 2 F_{r}(2,4)=\frac{i}{3}(k-1)(2 k-1)^{2}(2 k-3)-(2 k-3)\left(14 k^{2}-31 k+18\right) n_{1}^{2} \\
& +\left(74 k^{2}-229 k+180\right) n_{1}^{4}-(47 k-82) n_{1}^{6}+3 n_{1}^{8} \\
& 3 \mathrm{~F}_{\mathrm{n}}(0,7)=\frac{1}{105}(2 \mathrm{k}-1)(2 \mathrm{k}-3)(2 \mathrm{k}-5)-\frac{1}{5}(2 \mathrm{k}-3)(2 \mathrm{k}-5) \mathrm{n}_{1}{ }^{2}+(2 \mathrm{k}-5) \mathrm{n}_{1}{ }^{4}-\mathrm{n}_{1}{ }^{6} \\
& 3 P_{n}(1,6)=\frac{1}{30}(2 k-1)^{2}(2 k-3)(2 k-5)-\frac{2}{15}(2 k-3)(2 k-5)(13 k-14) n_{1}{ }^{2} \\
& +(6 k-15)(4 k-7) r_{1}{ }^{4}-(22 k-56) n_{1}^{6}+25 n_{1}^{8} \\
& 5 F_{r 1}(2,5)=\frac{1}{3}(k-1)(2 k-1)^{2}(2 k-3)-\frac{1}{3}(2 k-3)\left(58 k^{2}-135 k+83\right) n_{1}^{2} \\
& +\left(158 k^{2}-527 k+41\right) n_{1}^{4}-5(39 k-77) n_{1}^{6}+50 n_{1}^{8}
\end{aligned}
$$


$3 \mathrm{P}_{\mathrm{n}}(3,4)=\frac{1}{6}(\mathrm{k}-1)(2 \mathrm{k}-1)^{2}(2 \mathrm{k}-3)^{2}-\frac{1}{2}(2 \mathrm{k}-3)^{2}\left(20 \mathrm{k}^{2}-48 \mathrm{k}+31\right) \mathrm{n}_{1}^{2}+\left(172 \mathrm{k}^{3}\right.$ $\left.-852 k^{2}+1433 k-819\right) n_{1}^{4}-\left(232 k^{2}-866 k+819\right) n_{1}^{6}+\frac{3}{2}(49 k-93) n_{1}^{8}$ $-\frac{3}{2} n_{1} 10$

$6 \mathrm{P}_{\mathrm{n}}(0,8)=\frac{1}{420}(2 \mathrm{k}-1)(2 \mathrm{k}-3)(2 \mathrm{k}-5)(2 \mathrm{k}-7)-\frac{1}{15}(2 \mathrm{k}-3)(2 \mathrm{k}-5)(2 \mathrm{k}-7) \mathrm{n}_{1}{ }^{2}$ $+\frac{1}{2}(2 k-5)(2 k-7) n_{1}^{4}-(2 k-7) n_{1}^{6}+\frac{1}{4} n_{1}^{8}$

$105 \mathrm{P}_{\mathrm{n}}(1,7)=\frac{1}{3}(2 \mathrm{k}-1)^{2}(2 \mathrm{k}-3)(2 \mathrm{k}-5)-\frac{4}{3}(2 \mathrm{k}-3)(2 \mathrm{k}-5)(17 \mathrm{k}-19) \mathrm{n}_{1}{ }^{2}$ $+14(2 k-5)(16 k-29) n_{1}^{4}-20(35 k-91) n_{l}^{6}+245 n_{l}^{8}$

$90 P_{n}(2,6)=(k-1)(2 k-1)^{2}(2 k-3)(2 k-5)-2(2 k-3)(2 k-5)\left(38 k^{2}-92 k+59\right) n_{1}^{2}$ $+10(2 k-5)\left(88 k^{2}-305 k+273\right) r_{1} 1^{4}-60\left(59 k^{2}-276 k+322\right) r_{1}{ }^{6}$ $+15(137 k-361) n_{1}^{8}-150 n_{1} 10$

$15 \mathrm{P}_{\mathrm{n}}(3,5)=\frac{1}{3}(\mathrm{k}-1)(2 \mathrm{k}-1)^{2}(2 \mathrm{k}-3)^{2}-\frac{2}{3}(2 \mathrm{k}-3)^{2}\left(40 \mathrm{k}^{2}-102 \mathrm{k}+71\right) \mathrm{n}_{1}{ }^{2}$ $+2\left(332 k^{3}-1740 k^{2}+3121 k-1917\right) n_{1}^{4}-4\left(374 k^{2}-1543 k+1638\right) n_{1} 6$ $+25(43 k-99) n_{1}^{8}-150 n_{1} 10$

\section{DISCUSSIO::}

J. Saastamoinen: rerlied to a luestion from Garfink, tiat in he refraction calculations we need atmosphere models wit: a leas layers. Two layers models are not enougit. 